\title{
Mitteilungen des rtaustria
}

\section{Herzlichen Dank an Thieme für ein erweitertes Bildungsservice gemeinsam mit rtaustria}

CRTE wird erweitert um die CPD-Benennung bei der Bepunktung von Bildungsleistungen und bei der Wissensüberprüfung. Jedem Mitglied werden nach dem positiven Test die Punkte in das persönliche e-Portfolio übertragen. rtaustria und Thieme vollziehen damit den nächsten Schritt einer bereits seit Jahren hervorragenden Bildungspartnerschaft.

\section{Auszeichnung für e-Learning „Erstellerkurs" - COMENIUS AWARD}

Unser e-Learning „Mammographie Erstellerkurs“, welcher in der Kategorie DMP „Digitale Multimedia Projekte“ antrat, wurde von der Gesellschaft für Pädagogik, Information und Medien ausgezeichnet.

Das Bildungskonsortium besteht aus dem Autraggeber rtaustria/Learnlinked für digitale Bildungsinfrastrukur sowie Webducation als Contentproduzent. Webducation war Einreicher beim Award in der Kategorie „berufliche Fortbildung“. Dieses Produkt wurde mit dem EDUMedia-Siegel 2015 für ein exemplarisches Bildungsprojekt per Urkunde versehen. Wir bedanken uns bei unseren Partner learnlinked.com und Webducation für das gelungene Projekt.

rtaustria führt das ausgezeichnete Projekt mit seinen Partnern unter ständiger technischer Weiterentwicklung fort. Seit 2015 kann das e-Learning des „Mammographie Erstellerkurses“ mit allen Endgeräten aufgerufen und absolviert werden.

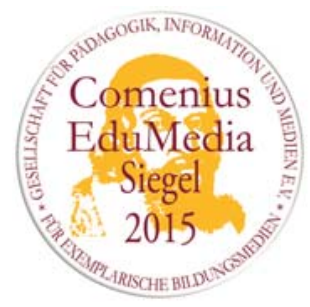

${ }^{\circ}$ Comenius EDUMedia

rtaustria-Fortbildung für Führungskräfte

Die ersten Teilnehmerinnen und Teilnehmer konnten erfolgreich alle 4 Module abschließen. Dazu gehörten ein allgemeines Modul, Modul Konfliktmanagement, Modul Prozessmanagement und Modul „Bewusst führen“.
Alle Teilnehmerinnen und Teilnehmer gaben uns jeweils sein sehr gutes Feedback und wir werden diese Weiterbildung fortsetzen.

Zielgruppen:

- Radiologietechnologen und -technologinnen in Leitungspositionen, wie Teamleitungen, Praxissupervisoren und -supervisorinnen (Praxisanleitungen), Bereichsleitungen pro Modalitäten

- Berufsangehörige der gehobenen MTD-Berufe

- Ärzte und Ärztinnen

- Physiker und Physikerinnen

Das ausgebuchte Modul „Bewusst führen“ fand am 27.Juni im Reitstall Radner, Neudörfl, statt. Die Teilnehmerinnen und Teilnehmer waren sehr engagiert bei der Sache. Unsere Co-Trainer „Como“ und „Santjo“ arbeiteten vorbildlich mit und waren sehr brav. Obwohl Respekt vor der Größe und dem Temperament der Pferde vorhanden war, gelangten alle Teilnehmerinnen und Teilnehmer zu unterschiedlichen Erkenntnissen gegenüber ihrer eigenen Persönlichkeit und Führungsfähigkeit.
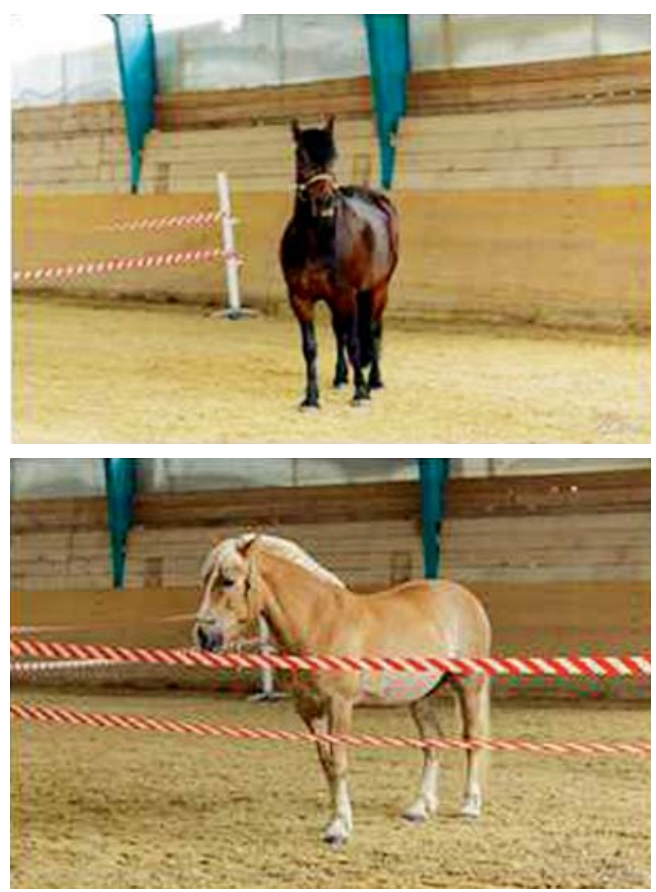

rtaustria-Kongress 2016 - Leitthemen „Strahlen \& Schutz“

\section{Vortragsprogramm}

Die Vorträge im Plenum am Freitag und Samstag werden die verschiedensten Aspekte der Leitthemen Strahlen \& Schutz abdecken. Auszug aus dem Vortragsprogramm (Änderungen vorbehalten):

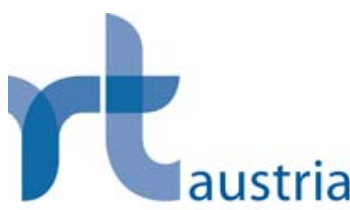

Como und Santjo bei „Bewusst führen“ ( ${ }^{\circ}$ rtaustria).

Geschäftsstelle:
rtaustria - Verband der
Radiologiechnologinnen
und -technologen
Österreichs
Johannes-Gutenberg-
Straße 3
2700 Wiener Neustadt
Österreich
Tel.: + 43 (0) 6641444060
Fax: +43 (0) 263877303
E-Mail:
office@radiologie-
technologen.at
Facebook:
www.facebook.com/
rtaustria

Geschäftsstelle: rtaustria - Verband de und -technologen Österreichs Straße 3 2700 Wiener Neustadt Österreich

E-Mail:

office@radiologie-

technologen.at

www.facebook.com/ rtaustria 


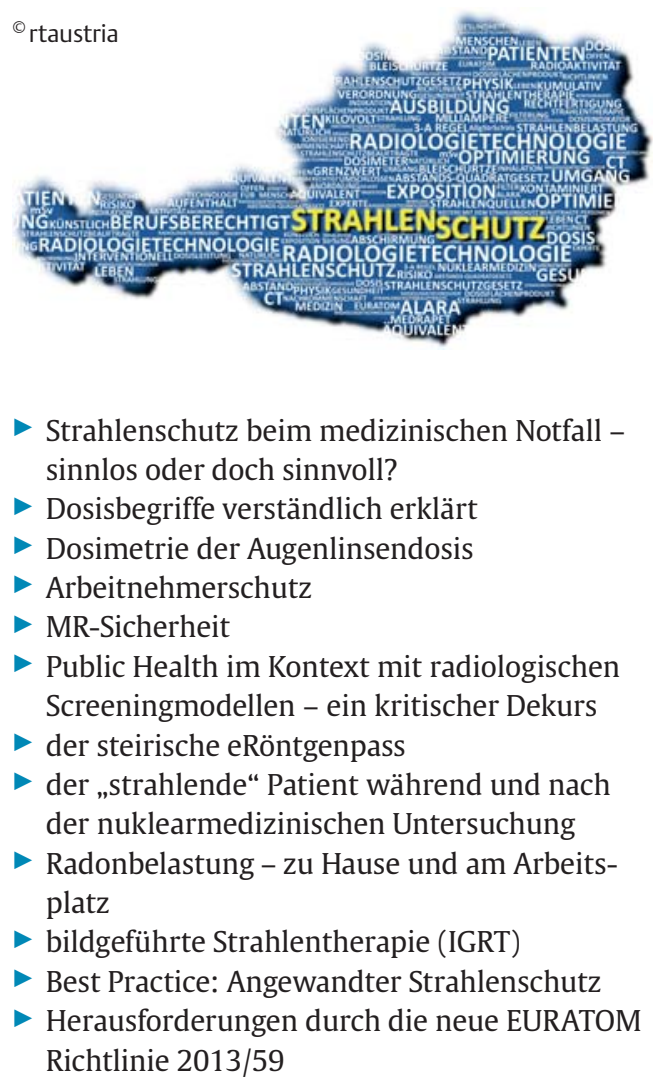

Veranstaltungsort: Steiermarkhof, Graz

21. 4. 2016: Abendsymposium, Eintritt frei, Der Österreichkongress für RadiologieAnmeldung auch ohne Kongressbesuch technologinnen und -technologen ist möglich

22.4. - 23.4.2016: Kongress mit Vor-

trägen im Plenum

22.4.2016: Abendveranstaltung „Feuerwerk für den Gaumen“

Am Samstag starten wir „Aktiv in den Tag“

mit dem Vitalcoach der Uniqa.

Extra 2 in 1: Kongress \& Erfüllung Fortbildungsverpflichtung Strahlenschutzgesetz

Mit dem Besuch des gesamten Kongresses schutzbeauftragte und weitere mit der wird gleichzeitig die gemäß §41 Abs. 4 All- Wahrnehmung des Strahlenschutzes begemeine Strahlenschutzverordnung vorge- traute Personen. Die Teilnehmer erhalten schriebene Fortbildungsverpflichtung im nach erfolgreicher Überprüfung der AnweAusmaß von 8 Stunden erbracht. Die Fort- senheit eine entsprechende Bestätigung. bildungsverpflichtung umfasst Strahlen-

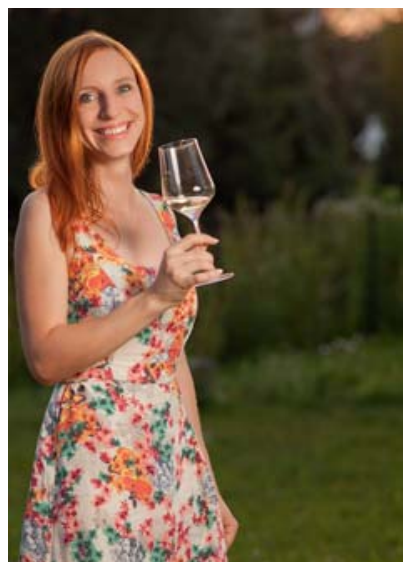

Diplom Sommelière Dominique Neuber $\left({ }^{\circ}\right.$ Photos by Markus Jöbstl).

nicht jede Weinkarte zieren. Koller, Schnabel, Grießbacher - noch nie gehört? Dann lassen Sie sich überraschen.

\section{2. rtaustria Posteraward: Call for Posters}

Im Rahmen des Kongresses veranstaltet rtaustria wieder einen Posteraward und ruft alle Radiologietechnologinnen und -technologen und Studierende auf, ihre wissenschaftlichen Untersuchungen als auch innovativen Projekte aus der klinischen Routine einzureichen.

Ziel ist es eine Plattform anzubieten, bei der die herausragenden Leistungen von Berufskolleginnen und -kollegen und Studierenden einem breiten Fachpublikum präsentiert werden können.

\section{Kongresspartner 2016}

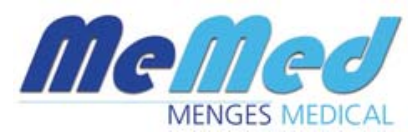

Folgende Unternehmen präsentieren sich als Aussteller oder mit interessanten Informationen.

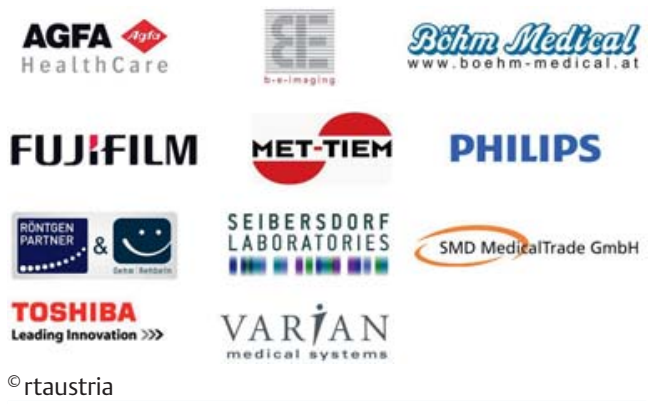

\section{Abendveranstaltung am 22.4.2016 - Feuerwerk für den Gaumen!}

Den ersten Kongresstag lassen wir mit einem delikaten 5-Gänge-Menü mit kommentierter Weinbegleitung ausklingen.

Wenige Gehminuten vom Kongressort entfernt findet das „Feuerwerk für den Gaumen“ beim „Lindenwirt“ statt. Das Küchenteam wird uns mit kulinarischen Hochgenüssen überraschen. Diplom Sommelière Dominique Neuber wird uns in die Welt der Weine entführen und für jeden Gang den passenden Begleiter vorstellen. Frau Neuber hat im Laufe der Zeit eine besondere Affinität zum steirischen Wein und vor allem für die kleinen aber feinen Weingüter entwickelt, die noch

Immer up-to-date zum Kongress 2016 sein:

Abonnieren Sie unseren Newsletter: www.radiologietechnologen.at/newsletter Besuchen Sie uns auf Facebook: https://de-de.facebook.com/RTaustria

\section{Neu im rtaustria-Kursangebot}

Detaillierte Information und Anmeldung: www.radiologietechnologen.at/fortbildungen/ anmelden/ 


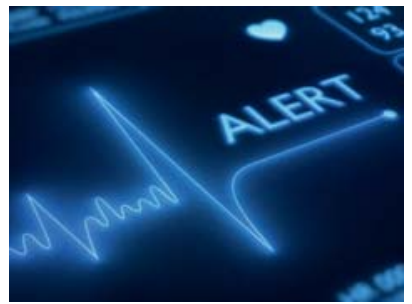

${ }^{0}$ Depositphotos/ JohanSwanepoel.

Basic Life-Support - Notfallmanagement „Sind sie gewappnet für den Notfall am Arbeitsplatz?"

Datum: 21. November 2015, Zeit: 9:00 - 16:30 Uhr Ort: Wien

Kosten: Mitglieder: $295 € /$ reguläre Teilnehmer: $395 €$

Zielgruppe: Radiologietechnologinnen und -technologen, MAB-RöASS, MTF, Ärzte und Ärztinnen; CPD- Bewertung: 24 CPD-Punkte inkl. pos. Wissensprüfung, 12 CPD-Punkte ohne Wissensprüfung

Kompetenzerwerb: kardiopulmonale Basis-Reaninmation nach int. Standard, situatives Notfallmanagement und Teamarbeit

Lehrinhalte:

Theoretische Inhalte:

Sicherheit vor Ort, Erkennen eines Ja-Sager-Patienten, Schwellenpatienten, Nein- Sager-Patienten, Notfalltherapie dieser Patientengruppen: Herzdruckmassage, stabile Seitenlage, Defibrillation, allgemeine Schockbekämpfung, situative Schocklagerung-Basismaßnahmen

Praktische Übungen:

Patientenbeurteilung, stabile Seitenlage, Herzdruckmassage, Beatmung, Defibrillation, allgemeine Schockbekämpfung, situative Schocklagerung-Basismaßnahmen

Lehr- und Lernformen: Vortrag, Gruppengespräch, Skill-Training, Reflexion

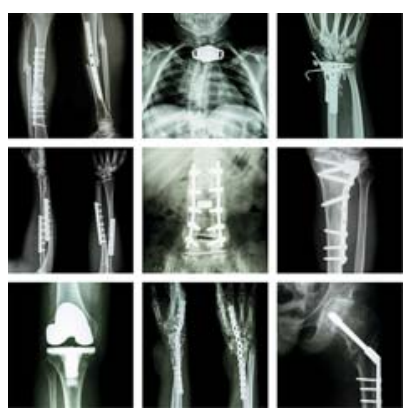

${ }^{\circ}$ Depositphotos/ stockdevil_666

„Unfallröntgen leicht gemacht"

Datum: 9.10.2015, Zeit: 8:30-17:00 Uhr

Ort: Wien

Kosten: rtaustria Mitglieder: $160 € /$ reguläre Teilnehmer: $230 €$

Zielgruppe: Radiologietechnologinnen und -technologen, MTF, MAB-RöAss.

Stundenumfang: 9 Unterrichtseinheiten, jeweils 45 Minuten

CPD-Bewertung: 18 CPD-Punkte inkl. pos. Wissensüberprüfung, 9 CPD-Punkte ohne Wissensüberprüfung
Kompetenzerwerb: Vertiefung- und/oder Spezialisierung im Bereich Unfallröntgen

Lehrinhalte:

Theoretische Inhalte:

Einstelltechnik in der Traumatologie, Bildanalyse: typische Pathologien, Strahlenschutz: Möglichkeiten der Dosisoptimierung, Stellenwert gut eingestellter Röntgenaufnahmen für die OP- und Behandlungsplanung

Praktische Übungen:

Strahlenschutz und Dosisoptimierung: praktische Anwendungen, Spezialaufnahmen bei Traumapatienten

Lehr- und Lernformen: Vortrag, Gruppengespräch, SkillTraining, Diskussion

\section{MTD-Forum in Kooperation mit \\ FH Campus Wien \\ GESUNDHEIT BRAUCHT BILDUNG}

international - interprofessionell - innovativ

Verleihung des MTD-Innovationspreises 2015

Freitag, 13. November 2015, 9:00-17:00 Uhr

Festsaal der FH Campus Wien

Treffen Sie Kolleginnen und Kollegen aus allen

7 Berufssparten zum Netzwerken.

Information und Anmeldung: www.mtd-austria. at

Wir freuen uns auf Ihren Besuch!
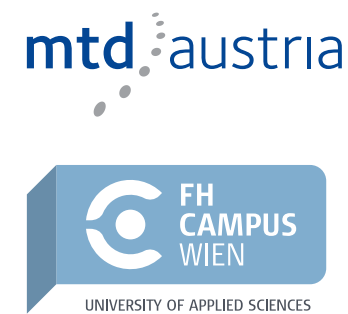

\section{Bericht in CIRS Medical Newsletter}

Im letzten Newsletter von CIRS medical gab es für unsere Berufsgruppe einen besonders interessanten Fall:

Ein Radiologietechnologe berichtete von einer Verwechslung zwischen dem unsterilen Kontrastmittel Peritrast ${ }^{\circledR}$ GI zum Einnehmen und dem sterilen Kontrastmittel Peritrast ${ }^{\circledR} 180$ bzw. 300 , welches intravenös injiziert oder in eine Körperhöhle eingebracht wird. Obwohl es eine Rücksprache mit dem Arzt/der Ärztin gab, wurde das unsterile Peritrast ${ }^{\circledR}$ GI im Rahmen einer retrograden Cystografie verabreicht. Ein Experte/eine Expertin von rtaustria gab viele Lösungsvorschläge, u.a. sollten Produkte mit gleicher Nomenklatur und unterschiedlicher Darreichungsform vermieden werden (z.B. Gastrografin ${ }^{\circledR}$ statt Peritrast ${ }^{\circledR}$ GI).

Neben der Verbesserung der internen Kommunikation und Überarbeitung der Arbeitsanweisung wird auf ein entsprechendes Stressmanagement verwiesen. Außerdem wird nochmals deutlich, dass unsere Berufsgruppe das nötige Fachwissen besitzt und dem Arzt/der Ärztin nicht blind vertrauen darf. Eine dezidierte Fallbeschreibung sowie eine entsprechende Fallanalyse finden Sie unter http://www.cirsmedical.at/, Fall 112328.

Tragen auch Sie Ihre Fälle im cirsmedical ein und leisten Sie einen Beitrag zur Patientensicherheit! 